\title{
LORENTZ BOOSTED NUCLEON-NUCLEON T-MATRIX AND THE TRITON BINDING ENERGY
}

\author{
H. KAMADA \\ Department of Physics, Faculty of Engineering, Kyushu Institute of Technology, \\ 1-1 Sensuicho Tobata, Kitakyushu 804-8550, Japan \\ kamada@mns.kyutech.ac.jp \\ W. GLÖCKLE \\ Institut für Theoretische Physik II, Ruhr-Universität Bochum, \\ D-44780 Bochum, Germany \\ H. WITAŁA, J. GOLAK, R. SKIBIŃSKI \\ M. Smoluchowski Institute of Physics, Jagiellonian University, PL-30059 Kraków, Poland \\ W. POLYZOU \\ Department of Physics and Astronomy, The University of Iowa, Iowa City, IA 52242, USA \\ CH. ELSTER \\ Institute of Nuclear and Particle Physics, and Department of Physics, Ohio University, Athens, \\ Ohio 45701, USA \\ Received (Day Month Year) \\ Revised (Day Month Year)
}

The phase equivalent relativistic NN potential, which is related by a nonlinear equation to the original nonrelativistic potential, is used to construct the mass operator (rest Hamiltonian) of the 3-nucleon system. Employing the CD Bonn NN potential, the solution of the relativistic $3 \mathrm{~N}$ Faddeev equation for ${ }^{3} \mathrm{H}$ shows slightly less binding energy than the corresponding nonrelativistic result. The effect of the Wigner spin rotation on the binding is very small.

Keywords: Relativity, Faddeev equation, Lorentz Boost

PACS Nos.:21.45.+v, 24.70.+s, 25.10.+s, 25.40.Lw

\section{Introduction}

Considerable experimental effort has been made in measuring proton-deuteron (pd) scattering $1|2| 3|4| 5$ cross sections at intermediate energies. For up to $300 \mathrm{MeV}$ proton energy those data have been analyzed with rigorous three-nucleon (3N) Faddeev calculations 6 based on the CD-Bonn potential 7 and the Tucson-Melbourne $3 \mathrm{~N}$ force $(3 \mathrm{NF}) 8$. Theoretical predictions based on $2 \mathrm{~N}$ forces alone are not sufficient 
to describe the data above about $100 \mathrm{MeV}$. Some of those defects are known as the Sagara discrepancy 9|10/11. Though 3NF effects are already seen below $100 \mathrm{MeV}$, they increase significantly above that energy. However, presently available 3NF's only partially improve the description of cross section data and spin observables. Since most of the cited calculations are based on the non-relativistic formulation of the Faddeev equations 12 , one needs to question if in the intermediate energy regime a Poincaré invariant formulation is more adequate.

There are two basic approaches to a relativistic formulation of the $3 \mathrm{~N}$ problem. One is a manifestly covariant scheme linked to a field theoretical approach 13 , the other is based on an exact realization of the symmetry of the Poincare group in three nucleon quantum mechanics 14 . We employ the second approach, where the mass operator (rest energy operator) consists of relativistic kinetic energies together with two- and many-body interactions including their boost corrections 15 .

The first attempt in solving the relativistic Faddeev equation for the $3 \mathrm{~N}$ bound state based on second approach has been carried out in 16, resulting in a decrease of the binding energy compared to the nonrelativistic result. On the other hand, similar calculations based on the field theory approach 13 increase it. These contradictory results require more investigation. In the following we summarize the results of our calculations based on the second approach: in Section 2 we introduce the relativistic $2 \mathrm{~N}$ potential, in Section 3 we present the $2 \mathrm{~N}$ t-matrix, which fulfills the relativistic boosted Lippmann-Schwinger (LS) equation, and in Section 4 we give numerical results for the triton binding energy based on the Poincaré invariant Faddeev equation.

\section{The Relativistic Potential}

Modern meson theoretical NN potentials, e.g. charge dependent Bonn Potential (CD-Bonn) 7, are derived from a relativistic Lagrangian, then cast into a threedimensional form using the Blankenbeclar-Sugar equation, which by kinematical redefinitions can be written in the form of a standard nonrelativistic LS equation, which in partial wave decomposed form reads

$$
t\left(p, p^{\prime} ; \frac{p^{\prime 2}}{m}\right)=v\left(p, p^{\prime}\right)+\int_{0}^{\infty} \frac{v\left(p, p^{\prime}\right) t\left(p^{\prime \prime}, p^{\prime} ; \frac{p^{2}}{m}\right)}{\frac{p^{\prime 2}}{m}-\frac{p^{\prime 2}}{m}+i \epsilon} p^{\prime \prime 2} d p^{\prime \prime} .
$$

The corresponding relativistic LS equation is given as

$$
t^{r}\left(p, p^{\prime} ; E\right)=v^{r}\left(p, p^{\prime}\right)+\int_{0}^{\infty} \frac{v^{r}\left(p, p^{\prime \prime}\right) t^{r}\left(p^{\prime \prime}, p^{\prime} ; E\right)}{E-2 \sqrt{{p^{\prime \prime}}^{2}+m^{2}}+i \epsilon} p^{\prime 2} d p^{\prime \prime}
$$

where

$$
E=2 \sqrt{p^{\prime 2}+m^{2}}
$$

In the relativistic Faddeev equation one needs $t^{r}$ off-the-energy-shell. According to 17 there is a direct operator relation between the nonrelativistic $v$ and the 
relativistic $v^{r}$ :

$$
4 m \hat{v}=2 \sqrt{\hat{p}^{2}+m^{2}} \hat{v}^{r}+2 \hat{v}^{r} \sqrt{\hat{p}^{2}+m^{2}}+\left(\hat{v}^{r}\right)^{2}
$$

In a momentum representation this leads to

$$
\begin{aligned}
& 4 m v\left(p, p^{\prime}\right)= \\
& v^{r}\left(p, p^{\prime}\right)\left(2 \sqrt{p^{2}+m^{2}}+2 \sqrt{p^{\prime 2}+m^{2}}\right)+\int_{0}^{\infty} d p^{\prime \prime} p^{\prime \prime 2} v^{r}\left(p, p^{\prime \prime}\right) v^{r}\left(p^{\prime \prime}, p^{\prime}\right) .
\end{aligned}
$$

This is the nonlinear relation between the relativistic potential $v^{r}$ and the nonrelativistic potential $v$ from Eq. (11), which has recently been solved 18 . The resulting on-shell-t-matrix $t^{r}$ is on-shell identical to the t-matrix $t$ from Eq. (1).

\section{The Lorentz Boosted T-matrix}

Cluster properties require that the energy is additive. Because of the non-linear relations between the mass and energy in special relativity, the additivity of energies in the rest frame implies a non-linear relation between the two-body interactions in the two and three-body mass operators 14. We call the two-body interaction in the three-body mass operator the "boosted potential".

$$
\hat{v}_{q}^{r} \equiv \sqrt{\left[2 \sqrt{\hat{p}^{2}+m^{2}}+\hat{v}^{r}\right]^{2}+q^{2}}-\sqrt{\left[2 \sqrt{\hat{p}^{2}+m^{2}}\right]^{2}+q^{2}},
$$

where the spectator momentum $q$ in the 3-body center of mass is simultaneously the negative total momentum of the pair. Using Eq. (44) this can be rewritten as $\frac{18}{18}$

$$
\begin{aligned}
4 m v\left(p, p^{\prime}\right) & =v_{q}^{r}\left(p, p^{\prime}\right)\left(\sqrt{4\left(p^{2}+m^{2}\right)+q^{2}}+\sqrt{4\left({p^{\prime}}^{2}+m^{2}\right)+q^{2}}\right) \\
& +\int_{0}^{\infty} d p^{\prime \prime} p^{\prime \prime 2} v_{q}^{r}\left(p, p^{\prime \prime}\right) v_{q}^{r}\left(p^{\prime \prime}, p^{\prime}\right) .
\end{aligned}
$$

Thus one can obtain $v_{q}^{r}$ by the same technique 18 as $v^{r}$. The boosted off-shell t-matrix is the solution of the LS equation

$$
\begin{aligned}
t_{q}^{r}\left(p, p^{\prime} ; E_{q}\right) & =v_{q}^{r}\left(p, p^{\prime}\right) \\
& +\int_{0}^{\infty} \frac{v_{q}^{r}\left(p, p^{\prime}\right) t_{q}^{r}\left(p^{\prime \prime}, p^{\prime} ; E_{q}\right)}{\sqrt{4\left(k^{2}+m^{2}\right)+q^{2}}-\sqrt{4\left(p^{\prime \prime 2}+m^{2}\right)+q^{2}}+i \epsilon} p^{\prime \prime} d p^{\prime \prime} .
\end{aligned}
$$

with $E_{q}=\sqrt{4\left(k^{2}+m^{2}\right)+q^{2}}$.

In Fig. 1 we display the boosted half-shell $\left(p^{\prime}=k\right)$ t-matrix of the CD-Bonn 7 potential at $E_{l a b}=350 \mathrm{MeV}$ for three different spectator momenta $q$. The magnitude of the t-matrix gradually decreases with increasing the boost momentum $q$. It can be shown $19 \mid 21$ that the half-shell t-matrices $t_{q}^{r}\left(p, p^{\prime}=k ; E_{k}\right)$ and $t^{r}\left(p, p^{\prime}=k ; E_{k}\right)$ are related by simple factors

$$
t_{q}^{r}\left(p, k ; E_{k}\right)=\frac{2 \sqrt{p^{2}+m^{2}}+2 \sqrt{k^{2}+m^{2}}}{\sqrt{4\left(p^{2}+m^{2}\right)+q^{2}}+\sqrt{4\left(k^{2}+m^{2}\right)+q^{2}}} t^{r}\left(p, k ; E_{k}\right) .
$$


Solving Eqs. (2) and (8) to obtain $t^{r}$ and $t_{q}^{r}$ independently we numerically confirmed the relation (91) with high precision. It is the factor on front of $t^{r}$ in the right hand side of Eq. (91) which attenuates the amplitude of the t-matrix with increasing $q$. It can also be shown 19 that the relativistic half-shell t-matrix $t^{r}$ is related to the corresponding nonrelativistic one via

$$
t^{r}\left(p, k ; E=2 \sqrt{k^{2}+m^{2}}\right)=\frac{4 m}{2 \sqrt{k^{2}+m^{2}}+2 \sqrt{p^{2}+m^{2}}} t\left(p, k ; k^{2} / m\right) .
$$

The explicit construction of first $v^{r}$ and then $t^{r}$ is equivalent to obtaining $t^{r}$ via resolvent equations as suggested in 19 and carried out in $20 \mid 21$.
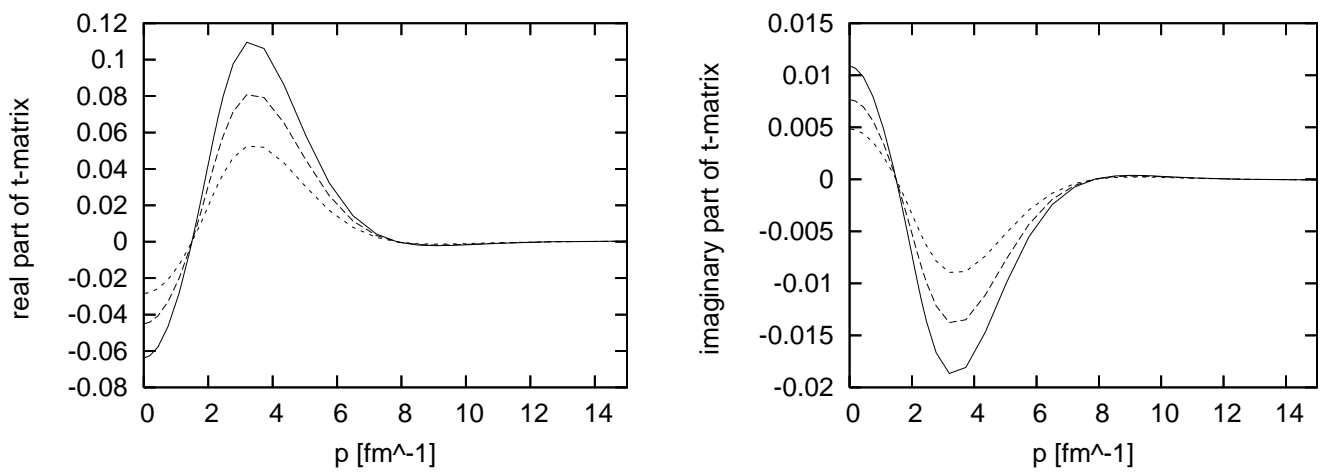

Fig. 1. The boosted half-on-the-mass-shell t-matrix of the CD-Bonn potential at $E_{l a b}=350 \mathrm{MeV}$. The left and right plots are real and imaginary parts, respectively. The solid, dashed and dotted lines are related to the boosting momentum $q=0,10$ and $20 \mathrm{fm}^{-1}$, respectively.

\section{The Triton Binding Energy}

The relativistic bound state Faddeev equation was solved using the boosted t-matrix $t_{q}^{r}$. In Table 1 the results for the triton binding energy using the CD-Bonn potential as input are shown. The triton binding energy obtained from the relativistic calculation is about $100 \mathrm{keV}$ smaller compared to the one calculated nonrelativistically. This value is significantly smaller than a previously published result 22 in which a reduction of the triton binding energy by about $400 \mathrm{keV}$ was given. The reason for this overestimation of a relativistic effect on the binding energy can be attributed to a different construction of the relativistic off-shell t-matrix $t^{r}$. The scaling transformation employed in 22 does not keep the $2 \mathrm{~N}$ scattering data invariant as function of the $2 \mathrm{~N}$ c.m. momentum.

We also included the Wigner spin rotation as outlined in 23 . Thereby the the Balian-Brezin method ${ }^{24}$ in handling the permutations is quite useful. In Table 2 the triton binding energies are shown allowing charge independence breaking (CIB) 26 and Wigner spin rotations. Wigner spin rotation effects reduce the binding energy by only about $2 \mathrm{keV}$. 
Table 1. The theoretical predictions of the trition binding energies resulting from the solutions of the nonrelativistic (first row) and relativistic (second row) Faddeev equations as function of the number of partial waves taken into account. The last line indicates the absolute difference between the nonrelativistic and relativistic result. In the calculations only the $\mathrm{np}$ force of the CD-Bonn potential was used. Unit is in $\mathrm{MeV}$.

\begin{tabular}{ccccc}
\hline & 5 ch $($ S-wave $)$ & $18 \operatorname{ch}\left(j_{\max }=2\right)$ & $26 \mathrm{ch}\left(j_{\max }=3\right)$ & $34 \mathrm{ch}\left(j_{\max }=4\right)$ \\
\hline nonrel. & -8.331 & -8.220 & -8.241 & -8.247 \\
rel. & -8.219 & -8.123 & -8.143 & -8.147 \\
diff. & 0.112 & 0.107 & 0.098 & 0.100 \\
\hline
\end{tabular}

Table 2. The theoretical predictions for the relativistic and nonrelativistic triton binding energies in $\mathrm{MeV}$. All numbers are 34 channels results. The second column is the same as the last column in Table 1 The results in the third column take charge dependence 26 into account. In addition the result of the fourth column contains also Wigner spin rotation effects.

\begin{tabular}{ccccc}
\hline & $\mathrm{np}$ force only & $\mathrm{np}+\mathrm{nn}$ forces & Wigner rotation & diff. \\
\hline nonrel. & -8.247 & -8.005 & - & - \\
rel. & -8.147 & -7.916 & -7.914 & -0.002 \\
diff. & 0.100 & 0.089 & - & - \\
\hline
\end{tabular}

\section{Summary and Outlook}

A phase-shift equivalent $2 \mathrm{~N}$ potential $\hat{v}^{r}$ in the relativistic $2 \mathrm{~N}$ Schrödinger equation is related to the potential $v$ in the nonrelativistic Schrödinger equation by the nonlinear relation given in Eq. (44). The boosted potential $\hat{v}_{q}^{r}$ is related to $\hat{v}^{r}$ by a similar expression, Eq. (6). With these potentials we generate the relativistic fully-off-shell t-matrix $t_{q}^{r}$, which enters into the relativistic Faddeev equation. We solve the relativistic bound state Faddeev equation and compare the binding energy for the triton with the one obtained from a nonrelativistic calculation with the same input interaction. We find that the difference between the two calculations is only about $90 \mathrm{keV}$ including CIB, where the relativistic calculation gives slightly less binding. Taking Wigner spin rotations into account in the relativistic calculation reduces the binding energy by a very small amount, $\approx 2 \mathrm{keV}$, indicating that Wigner rotations of the spin have essentially no effect on the predicted value of the binding energy.

Applications to the 3-body continuum are in progress. Recently 23 the formulation lined out above has been used to study the low energy $A_{y}$ puzzle in neutron-deuteron scattering. Details are presented by Witała in this conference. In the intermediate energy regime the formulation has been applied to exclusive proton-deuteron scattering cross sections at $508 \mathrm{MeV} 20 \mid 21$ based on a formulation of of the Faddeev equations which does not employ a partial wave decomposition. The approach can also be extended and applied to electromagnetic processes $27 / 28$. 


\section{Acknowledgments}

This work was partially supported by the 2008-2011 polish science funds as a research project No. N N202 077435. It was also partially supported by the Helmholtz Association through funds provided to the virtual institute "Spin and strong QCD"(VH-VI-231). The numerical calculations were performed on the IBM Regatta p690+ of the NIC in Jülich, Germany.

\section{References}

1. W. P. Abfalterer et al. Phys. Rev. Lett. 81, 57 (1998).

2. K. Sekiguchi et al. Phys. Rev. C 65, 034003 (2002).

3. K. Hatanaka et al. Phys. Rev. C 66, 044002 (2002).

4. K. Ermisch et al. Phys. Rev. C 71, 064004 (2005).

5. St. Kistryn et al. Phys. Rev. C 72, 044006 (2005).

6. H. Witała et al. Phys. Rev. C 59, 3035 (1999).

7. R. Machleidt, F. Sammarruca, Y. Song, Phys. Rev. C 53, R1483 (1996).

8. S. A. Coon, W. Glöckle, Phys. Rev. C 23, 1790 (1981).

9. K. Sagara et al. Phys. Rev. C 50, 576 (1994).

10. S. Nemoto et al. Phys. Rev. C 58, 2599 (1998).

11. H. Witała et al. Phys. Rev. Lett. 81, 1183 (1998).

12. W. Glöckle et al. Phys. Rep. 274, 107 (1996).

13. Alfred Stadler, Franz Gross, and Michael Frank Phys. Rev. C 56, 2396 (1997).

14. F. Coester, Helv. Phys. Acta 38, 7 (1965).

15. B. Bakamjian, L. H. Thomas, Phys. Rev. 92, 1300 (1953).

16. W. Glöckle, T-S. H. Lee, and F. Coester, Phys. Rev. C 33, 709 (1986).

17. F. Coester, S. C. Pieper, and F. J. D. Serduke, Phys. Rev. C 11, 1 (1975).

18. H. Kamada and W. Glöckle, Phys. Lett. B655, 119 (2007).

19. B. D. Keister and W.N. Polyzou, Phys. Rev. C 73, 014005 (2006).

20. T. Lin, Ch. Elster, W. N. Polyzou and W. Glöckle, Phys. Lett. B 660, 345 (2008).

21. T. Lin, Ch. Elster, W. N. Polyzou, H. Witała and W. Glöckle, Phys. Rev. C 78, 024002 (2008).

22. H. Kamada, W. Glöckle, J. Golak, Ch. Elster, Phys. Rev. C 66, 044010 (2002).

23. H. Witała, J. Golak, W. Glöckle, H. Kamada, W. N. Polyzou, Phys. Rev. C 77, 034004 (2008).

24. R. Balian et al. Il Nuovo Cim. B2, 403 (1969).

25. T. Lin, Ch. Elster, W. N. Polyzou and W. Glöckle, Phys. Rev. C 76, 014010 (2007).

26. H. Witała, W. Glöckle, H. Kamada, Phys. Rev. C 43, 1619 (1991).

27. J. Golak et al. Phys. Rep. 415, 89 (2005).

28. J. Golak, R. Skibiński, H. Witała, W. Glöckle, A. Nogga, H. Kamada, Acta Phys. Pol. $B$ 38, 2143 (2007). 